EPJ Web of Conferences 69, 00014 (2014)

DOI: $10.1051 /$ epjconf / 20146900014

(C) Owned by the authors, published by EDP Sciences, 2014

\title{
Towards the fusion of weakly bound projectiles with heavy tar- gets with proximity based potentials
}

\author{
Raj Kumari ${ }^{1, a}$ \\ ${ }^{1}$ Department of Physics, Panjab University, Chandigarh -160 014, India.
}

\begin{abstract}
Theoretical calculations using proximity based potentials are performed to predict the fusion cross sections of the reactions of weakly bound projectiles especially halo nuclei with ${ }^{209} \mathrm{Bi}$ target. Both neutron halo as well as proton halo projectiles are taken in the present analysis. In particular, we study the role of extended nuclear radii of these weakly bound nuclei on the fusion cross sections. The inability of ${ }^{8} \mathrm{~B}+{ }^{58} \mathrm{Ni}$ reaction to follow the systematics at above barrier energies motivates us to investigate the trend for the reactions of those weakly bound nuclei for which experiments are not yet performed. Our results indicate that extended radii of these weakly bound nuclei significantly affect fusion barrier heights as well as fusion probabilities throughout the energy range. We also study the fusion probabilities of a few reactions involving tightly bound projectiles.
\end{abstract}

\section{Introduction}

It is well known that the excitation energy determines the fate of a reaction. Low incident energies drive the reacting partners into complete (or incomplete) fusion followed by the pre-equilibrium emission of the nucleons [1]. Further increase in the excitation energy breaks colliding nuclei into many pieces [2]. Excitation energy of a reaction is also crucial to predict the nature of collective flow [3].

The pursuit of sub-barrier fusion has earned unique attention in the recent past in the reactions involving tightly bound as well as weakly bound nuclei. This renewed interest is also due to the availability of radioactive ion beams that lead to the formation of very neutron rich nuclei [4]. A number of experiments have been performed at sub-barrier energies [5] accompanied by efforts on the theoretical front [6] also. As reported by Balantekin and Takigawa [7], sub-barrier fusion reactions are not only important for stellar energy production and nucleosynthesis, but are also important for studying reaction dynamics and nuclear structure. The weakly bound nuclei, especially halo nuclei due to their extended radii and low energy threshold for break-up, have been a subject of great interest in the last few years. Most of the reactions involving these nuclei are found to follow the systematic behavior of fusion suppression above the barrier and enhancement at sub-barrier energies [8]. The reaction of the proton halo ${ }^{8} \mathrm{~B}$ projectile with a ${ }^{28} \mathrm{Si}$ target is found to follow the systematics [9], but in the case of ${ }^{8} \mathrm{~B}+{ }^{58} \mathrm{Ni}$ reaction, unexpected results are observed i.e. enhancement is observed at all energies $[9,10]$. This behavior differs from the systematics observed so far for all other weakly bound nuclei. Since different behaviors of proton halo and neutron halo projectiles [10], and even the same proton halo projectile with different targets [9], are observed towards fusion, it is very important to

a e-mail: rajkumari80pu@gmail.com

This is an Open Access article distributed under the terms of the Creative Commons Attribution License 2.0, which permits unrestricted use, distribution, and reproduction in any medium, provided the original work is properly cited. 
investigate the trends for other weakly bound nuclei for which experimental studies have not yet been made.

In Ref. [11], Dutt and Puri carried out a massive hunt for the best nucleon-nucleon potential by carefully analyzing about a dozen proximity based potentials, robust for a large number of empirical fusion barriers. There, the potentials due to AW 95, Bass 80 and Denisov DP were found to reproduce barrier heights and positions far better than others in the class [11]. In addition to many extensions of the original proximity potential, Dutt and Bansal [12] also proposed a new form of proximity potential (labelled as Prox. 2010). Apart from the proximity based potentials, one due to the Skyrme energy density formalism (SEDF) with Skyrme force SIII was also found to explain experimental barriers nicely [13]. The above comparison was limited to the reactions of tightly bound projectiles only. In some cases, mainly the above barrier region was considered only [13]. One is therefore, interested to test these potentials for the fusion of weakly bound projectiles with heavy targets. In Section 2, we describe the models, which is followed by the results in Section 3.

\section{Description of Models}

As noted above, a careful analysis of $[11,12]$ has shown that the potentials due to AW 95, Bass 80 , Denisov DP and Proximity 2010 yield close agreement with experimental data. All these potentials were parameterized in terms of the proximity concept $[12,14-16]$.

The proximity potential is based on the theorem that states that "the force between two gently curved surfaces in close proximity is proportional to the interaction potential per unit area between two flat surfaces" [17]. The interaction potential $V_{N}(R)$ between two colliding surfaces therefore, is given by

$$
V_{N}=4 \pi \gamma b \bar{C} \Phi\left(\frac{R-C_{1}-C_{2}}{b}\right) M e V
$$

where $\bar{C}\left(=\left(C_{1} C_{2}\right) /\left(C_{1}+C_{2}\right)\right), b$ and $R$ are the reduced radius, surface width and central separation, respectively. Here $\mathrm{C}$ is the central radius and $\Phi$ is the universal nuclear function. The surface energy coefficient $\gamma$ has the form

$$
\gamma=\gamma_{0}\left[1-k_{s}\left(\frac{N-Z}{A}\right)^{2}\right]
$$

Here $\gamma_{0}$ and $k_{s}$ are the surface energy coefficient and surface asymmetry constant, respectively. Though the proximity 1977 potential has been the benchmark for all the calculations, the recent form and value of $\gamma$, radius as well as $\Phi$ have lead to proximity 2010 [12]. Based on the liquid drop model, Bass had suggested a potential that can be parameterized in terms of the proximity concept [14]. A similar attempt was also made by A. Winther in his work [16]. On the other hand, Denisov performed calculations for 119 spherical or non-spherical even-even nuclei around the $\beta$-stability line and derived an expression for the nuclear part of the potential [15]. This potential was further improved by Dutt and Puri [11].

The potential based on the Skyrme energy density formalism (SEDF) was also solved within the proximity concept $[13,18]$. There, the Skyrme potential corresponding to SIII Skyrme force was found to reproduce barrier heights and positions accurately [13]. In this model, the nuclear part of the interaction potential $V_{N}(\mathrm{R})$ is defined as the difference between the energy expectation value $\mathrm{E}$ of two colliding nuclei at a finite distance $\mathrm{R}$ and at infinity :

$$
V_{N}(R)=E(R)-E(\infty),
$$


Table 1. Fusion barrier heights $V_{B}(\mathrm{MeV})$ for the reactions of tightly bound projectiles with heavy targets using AW 95, Bass 80, Denisov DP, Proximity 2010 and SEDF. The corresponding empirical values are also listed.

\begin{tabular}{|c|c|c|c|c|c|c|c|}
\hline Reaction & 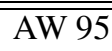 & 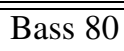 & "Denisov DP & Prox. 2010 & SEDF & Empirical & Ref. \\
\hline \multirow[t]{2}{*}{${ }^{28} \mathrm{Si}+{ }^{58} \mathrm{Ni}$} & 53.29 & 53.21 & 53.07 & 53.87 & 52.67 & 53.80 & {$[21]$} \\
\hline & & & & & & $\begin{array}{l} \pm 0.8 \\
52.90\end{array}$ & $\begin{array}{l}{[21]} \\
{[22]}\end{array}$ \\
\hline \multirow[t]{3}{*}{${ }^{30} \mathrm{Si}+{ }^{58} \mathrm{Ni}$} & 52.72 & 52.61 & 52.38 & 53.36 & 52.10 & 52.20 & \\
\hline & & & & & & \pm 1.2 & [21] \\
\hline & & & & & & 52.80 & [22] \\
\hline \multirow[t]{3}{*}{${ }^{30} \mathrm{Si}+{ }^{62} \mathrm{Ni}$} & 52.09 & 51.93 & 51.69 & 52.81 & 51.50 & 52.20 & \\
\hline & & & & & & \pm 0.9 & [21] \\
\hline & & & & & & 52.10 & [22] \\
\hline \multirow[t]{3}{*}{${ }^{30} \mathrm{Si}+{ }^{64} \mathrm{Ni}$} & 51.80 & 51.61 & 51.36 & 52.55 & 51.22 & 51.20 & \\
\hline & & & & & & \pm 0.9 & [21] \\
\hline & & & & & & 51.40 & [22] \\
\hline \multirow[t]{3}{*}{${ }^{32} \mathrm{~S}+{ }^{58} \mathrm{Ni}$} & 60.22 & 60.14 & 60.10 & 60.82 & 59.70 & 59.80 & \\
\hline & & & & & & \pm 1.4 & [21] \\
\hline & & & & & & 59.60 & [22] \\
\hline \multirow[t]{3}{*}{${ }^{34} \mathrm{~S}+{ }^{58} \mathrm{Ni}$} & 59.64 & 59.52 & 59.42 & 60.31 & 59.05 & 58.40 & \\
\hline & & & & & & \pm 1.4 & [21] \\
\hline & & & & & & 58.50 & [22] \\
\hline \multirow[t]{2}{*}{${ }^{36} \mathrm{~S}+{ }^{48} \mathrm{Ca}$} & 42.69 & 42.48 & 42.11 & 43.46 & 42.06 & 43.30 & [23] \\
\hline & & & & & & 43.30 & [24] \\
\hline \multirow[t]{4}{*}{${ }^{48} \mathrm{Ca}+{ }^{48} \mathrm{Ca}$} & 51.74 & 51.39 & 51.13 & 52.60 & 51.05 & 51.70 & {$[25]$} \\
\hline & & & & & & 51.49 & [27] \\
\hline & & & & & & 51.20 & [22] \\
\hline & & & & & & 51.90 & [24] \\
\hline
\end{tabular}

The energy E at infinity represents the binding energy of a nucleus in isolation. The energy expectation value $\mathrm{E}$ is given by

$$
E=\int H(\vec{r}) d \vec{r}
$$

The energy density function $\mathrm{H}$ is a function of nucleonic density $\rho$, kinetic energy density $\tau$ and spin density $\vec{J}$. The nuclear potential $V_{N}(R)$ is sum of a spin-independent part $V_{P}(R)$ and a spin-dependent part $V_{J}(R)$. We use Skyrme force SIII in the present study. For the details of all potentials, the reader is referred to Refs. [11-13]. By adding the Coulomb potential to the nuclear potential, one can compute the total potential $V_{T}(\mathrm{R})$ and hence barrier positions and heights.

For the accurate calculation and reproduction of the fusion cross-sections above the barrier, one should be able to reproduce the barrier height and position accurately. In contrast, the shape of the barrier is the most crucial parameter for the sub-barrier studies. The sub-barrier distribution can be computed within the Wong model [19].

Accordingly, the fusion cross section for complete fusion is given by

$$
\sigma_{f u s}\left(E_{c . m .}\right)=\frac{\pi}{k^{2}} \sum_{l=0}^{l_{\max }}(2 l+1) T_{l}\left(E_{c . m .}\right),
$$




\section{EPJ Web of Conferences}

Table 2. Fusion barrier positions $R_{B}(\mathrm{fm})$ for the reactions of tightly bound projectiles with heavy targets using AW 95, Bass 80, Denisov DP, Proximity 2010 and SEDF. The corresponding empirical values are also listed.

\begin{tabular}{|c|c|c|c|c|c|c|c|}
\hline Reaction & AW 95 & Bass 80 & Denisov DP & Prox. 2010 & $\overline{\text { SEDF }}$ & Empirical & Ref. \\
\hline \multirow[t]{3}{*}{${ }^{28} \mathrm{Si}+{ }^{58} \mathrm{Ni}$} & 9.86 & 9.78 & 9.78 & 9.69 & 9.73 & 9.00 & \\
\hline & & & & & & \pm 0.9 & [21] \\
\hline & & & & & & 8.10 & [22] \\
\hline \multirow[t]{3}{*}{${ }^{30} \mathrm{Si}+{ }^{58} \mathrm{Ni}$} & 9.94 & 9.92 & 9.96 & 9.82 & 9.88 & 8.30 & \\
\hline & & & & & & \pm 1.1 & [21] \\
\hline & & & & & & 8.80 & [22] \\
\hline \multirow{3}{*}{${ }^{30} \mathrm{Si}+{ }^{62} \mathrm{Ni}$} & 10.10 & 10.08 & 10.09 & 9.92 & 10.00 & 9.70 & \\
\hline & & & & & & \pm 1.0 & [21] \\
\hline & & & & & & 9.70 & [22] \\
\hline \multirow{3}{*}{${ }^{30} \mathrm{Si}+{ }^{64} \mathrm{Ni}$} & 10.15 & 10.14 & 10.15 & 9.97 & 10.09 & 9.40 & \\
\hline & & & & & & \pm 0.8 & [21] \\
\hline & & & & & & 9.60 & [22] \\
\hline \multirow{3}{*}{${ }^{32} \mathrm{~S}+{ }^{58} \mathrm{Ni}$} & 9.97 & 9.91 & 9.90 & 9.83 & 9.84 & 8.60 & \\
\hline & & & & & & \pm 0.9 & [21] \\
\hline & & & & & & 8.30 & [22] \\
\hline \multirow[t]{3}{*}{${ }^{34} \mathrm{~S}+{ }^{58} \mathrm{Ni}$} & 10.05 & 10.04 & 10.01 & 9.95 & 9.99 & 7.50 & \\
\hline & & & & & & \pm 0.9 & [21] \\
\hline & & & & & & 7.60 & [22] \\
\hline${ }^{36} \mathrm{~S}+{ }^{48} \mathrm{Ca}$ & 10.04 & 10.07 & 10.09 & 9.83 & 10.12 & 9.54 & [24] \\
\hline \multirow[t]{4}{*}{${ }^{48} \mathrm{Ca}+{ }^{48} \mathrm{Ca}$} & 10.39 & 10.40 & 10.42 & 10.16 & 10.40 & 10.38 & [25] \\
\hline & & & & & & 10.16 & [27] \\
\hline & & & & & & 11.20 & [22] \\
\hline & & & & & & 10.03 & [24] \\
\hline
\end{tabular}

where $l_{\max }$ corresponds to the largest partial wave for which a pocket still exists in the interaction potential and $T_{l}\left(E_{c . m .}\right)$ is the energy-dependent barrier penetration factor, approximated by the HillWheeler formula for a parabolic interaction barrier as:

$$
T_{l}\left(E_{c . m .}\right)=\left[1+\exp \left\{\frac{2 \pi}{\hbar \omega_{l}}\left(V_{B}^{l}\left(R_{l}\right)-E_{c . m .}\right)\right\}\right]^{-1},
$$

where $V_{B}^{l}\left(R_{l}\right)$ and $\hbar \omega_{l}$ are the fusion barrier heights and curvature, respectively, the for $l^{\text {th }}$ partial wave. Here, $R_{l}$ and $\hbar \omega_{l}$ are insensitive to $l$ and are therefore given by

$$
R_{l} \simeq R_{0}, \hbar \omega_{l} \simeq \hbar \omega_{0}
$$

and

$$
V_{B}^{l} \cong V_{B}^{0}+\frac{\hbar^{2} l(l+1)}{2 \mu R_{0}^{2}} .
$$

Using the above assumptions and replacing the summation in Eq. (5) by an integral results in

$$
\sigma_{f u s}(m b)=10 \frac{R_{B}^{2} \hbar \omega_{0}}{2 E_{c . m .}} \ln \left[1+\exp \left\{\frac{2 \pi}{\hbar \omega_{0}}\left(E_{c . m .}-V_{B}\right)\right\}\right]
$$




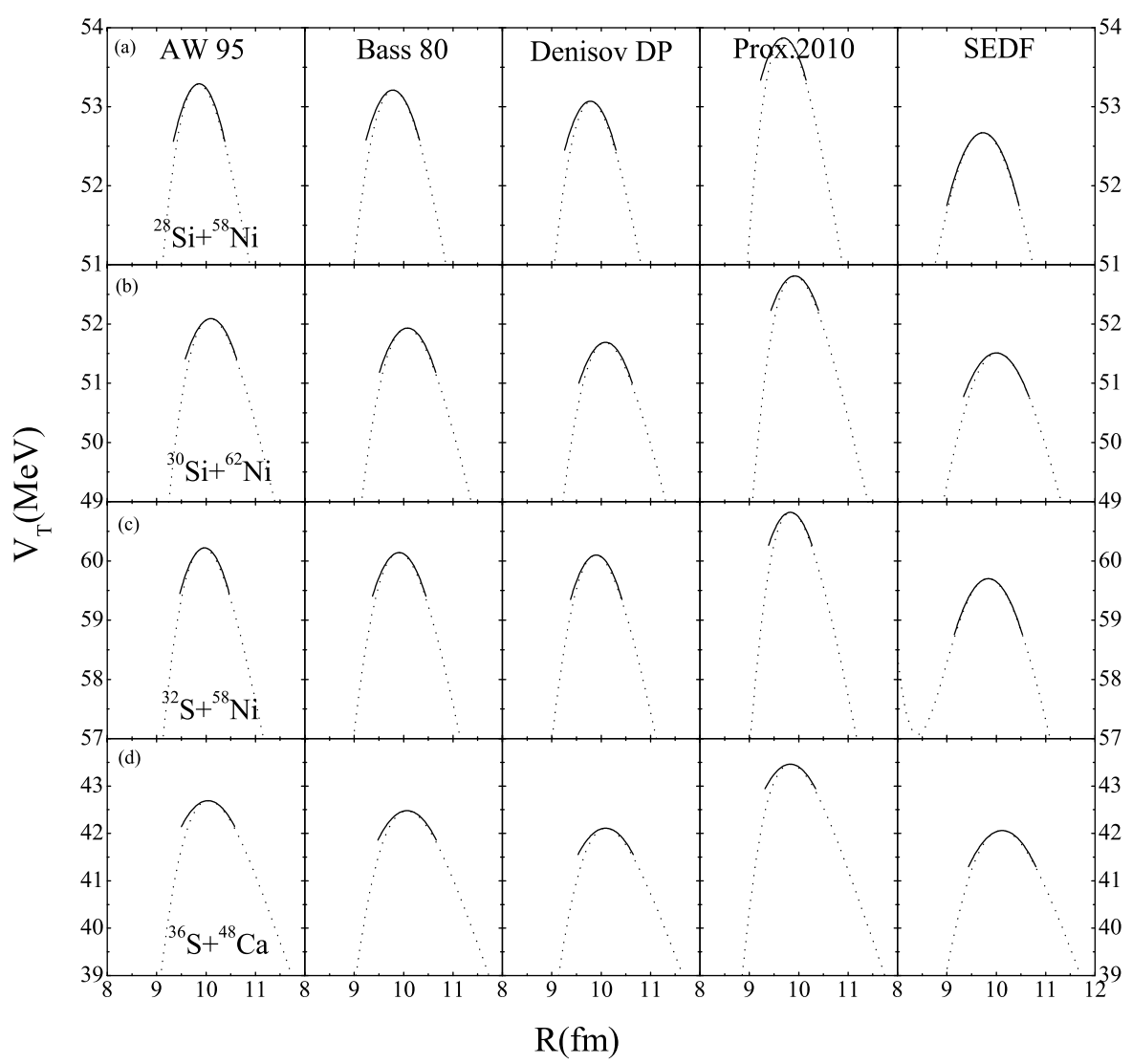

Figure 1. Total interaction potential $V_{T}(\mathrm{MeV})$ as a function of the internuclear distance $\mathrm{R}(\mathrm{fm})$ for the reactions of ${ }^{28} \mathrm{Si}+{ }^{58} \mathrm{Ni}$ (first row), ${ }^{30} \mathrm{Si}+{ }^{62} \mathrm{Ni}$ (second row) and ${ }^{32} \mathrm{~S}+{ }^{58} \mathrm{Ni}$ (third row) and ${ }^{36} \mathrm{~S}+{ }^{48} \mathrm{Ca}$ (fourth row). The solid lines indicate the harmonic oscillator inverse fit to the curve.

The fusion cross sections for the reactions of tightly bound projectiles is calculated using Eq. (9). But the above mentioned parametrization is inadequate to account for the total fusion cross-sections of the reactions involving weakly bound projectiles. Therefore, a new parameterized form given in Ref. [20] is used. The reduced energy is given by dividing the center of mass energy by $\frac{Z_{P} Z_{T}}{A_{P}^{1 / 3}+A_{T}^{1 / 3}}$ and the reduced cross-section is obtained by dividing the total reaction cross-section by the square of the radius $R=A_{P}^{1 / 3}+A_{T}^{1 / 3}$ ), where $A_{P}$ and $A_{T}$ are the mass numbers of the the projectile and target and $Z_{P}$ and $Z_{T}$ are their charges, respectively. 


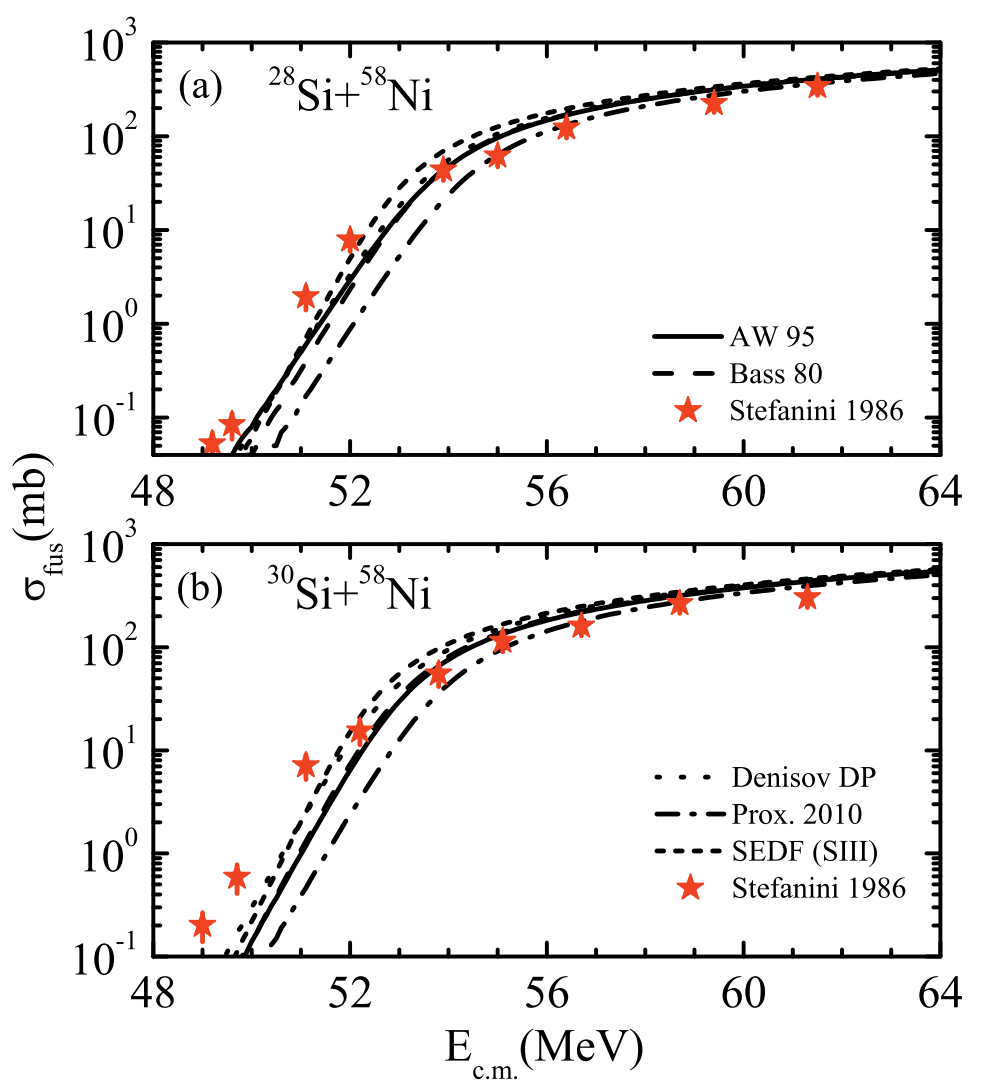

Figure 2. The fusion cross sections $\sigma_{\text {fus }}(\mathrm{mb})$ as a function of the center of mass energy $\mathrm{E}_{\mathrm{c} . \mathrm{m} .}(\mathrm{MeV})$ for the reactions of ${ }^{28} \mathrm{Si}+{ }^{58} \mathrm{Ni}$ and ${ }^{30} \mathrm{Si}+{ }^{58} \mathrm{Ni}$. The experimental data are taken from Stefanini 1986 [21].

\section{Result and Discussions}

For the present systematic study, we first calculated the fusion probabilities of a few reactions of tightly bound projectiles with heavy targets and then for the reactions of weakly bound projectiles with heavy targets.

\subsection{Fusion of tightly bound projectiles with heavy targets}

For the present systematic study, we calculated the fusion probabilities of the reactions of ${ }^{28,30} \mathrm{Si}+{ }^{58} \mathrm{Ni}$, ${ }^{30} \mathrm{Si}+{ }^{62,64} \mathrm{Ni},{ }^{32,34} \mathrm{~S}+{ }^{58} \mathrm{Ni},{ }^{36} \mathrm{~S}+{ }^{48} \mathrm{Ca}$ and ${ }^{48} \mathrm{Ca}+{ }^{48} \mathrm{Ca}$. In Table 1 , the calculated fusion barrier heights and, in table 2, the barrier positions are displayed for the above mentioned reactions using the potentials due to AW 95, Bass 80, Denisov DP, Proximity 2010 and SEDF (SIII force) along with the empirical values. From the table, it is evident that all potentials can reproduce the experimental barriers quite accurately. This also further justifies that these potentials are better than others available in the literature. As noted by many authors, the neutron content affects the barrier height significantly. 


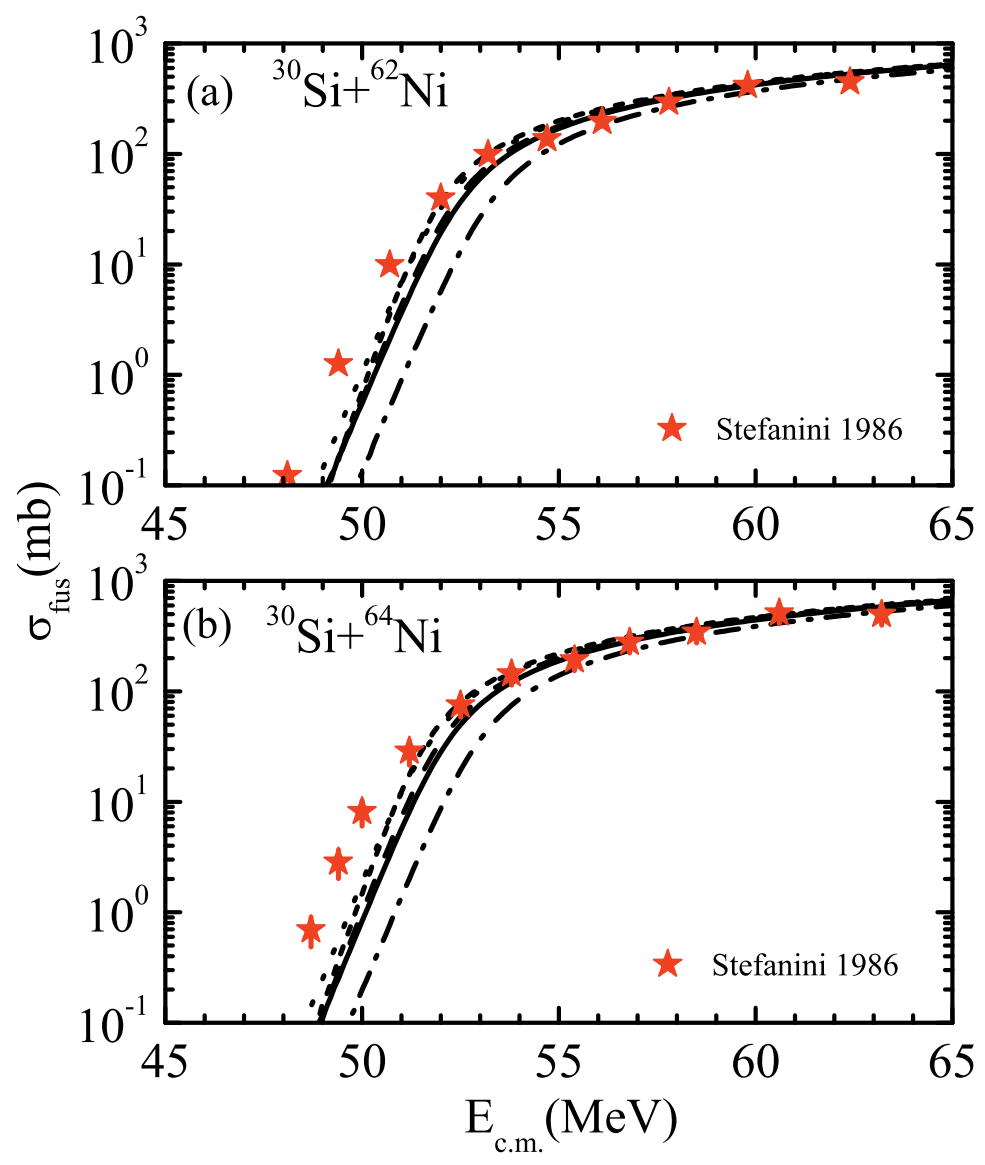

Figure 3. Same as fig. 2, but for the reactions of ${ }^{30} \mathrm{Si}+{ }^{62} \mathrm{Ni}$ and ${ }^{30} \mathrm{Si}+{ }^{64} \mathrm{Ni}$. The experimental data are taken from Stefanini 1986 [21].

From Table 1, we observe that as the neutron content of a reaction increases, the barrier height decreases. This trend remains the same if one keeps the target or projectile fixed. Further enhancement in the barrier height is seen with the mass of the colliding pair.

We display in Fig. 1, the potentials around the barrier and fit them with an inverted harmonic oscillator. The parameters of the inverted harmonic oscillator are then used in the Wong formula to calculate the fusion cross-sections. For the sub-barrier fusion, the shape of the potential and its parametrization is the most crucial one. It is evident that independent of the reacting partners, the barrier region can be fitted nicely by an inverted harmonic oscillator.

In Figs. 2-5, we display the fusion excitation functions $\sigma_{\text {fus }}(\mathrm{mb})$ calculated using the Wong formula as a function of center of mass energy, $\mathrm{E}_{\mathrm{c} . \mathrm{m} .}(\mathrm{MeV})$. The solid, dashed, dotted, dash-dotted and short dashed lines represent the calculations for AW 95, Bass 80, Denisov DP, Proximity 2010 and SEDF respectively. The stars represent the experimental data. 


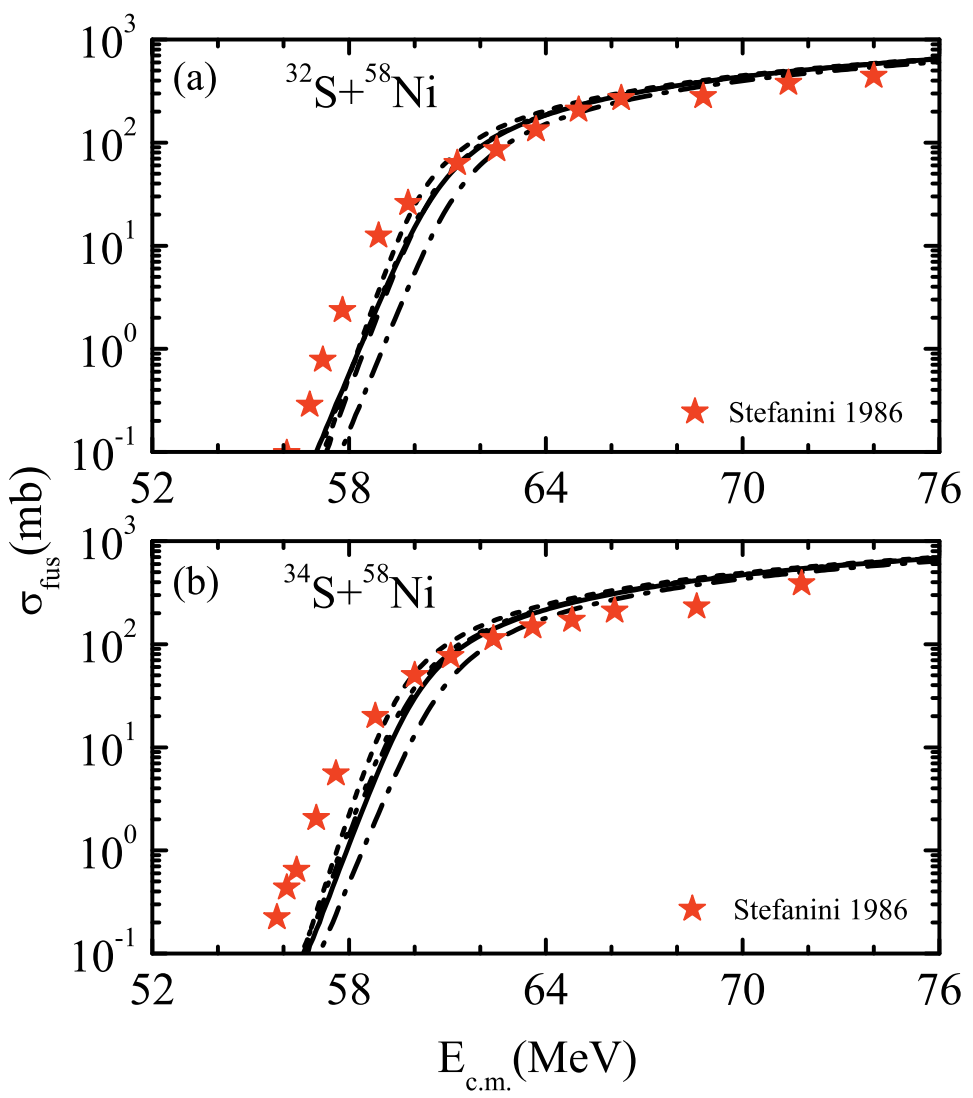

Figure 4. Same as fig. 2, but for the reactions of ${ }^{32} \mathrm{~S}+{ }^{58} \mathrm{Ni}$ and ${ }^{34} \mathrm{~S}+{ }^{58} \mathrm{Ni}$. The experimental data are taken from Stefanini 1986 [21].

From Figs. 2-5, it is evident that all of the proximity based potentials explain nicely the fusion cross-sections at above-barrier energies and a very small enhancement at sub-barrier energies is observed for the above mentioned reactions of tightly bound projectiles with heavy targets.

\subsection{Fusion of weakly bound projectiles with heavy targets}

In our recent study of fusion probabilities with halo nuclei [10], we found that the effect of extended size on the fusion probabilities of a few known reactions of halo projectiles strongly depends upon the nature of the halo nucleus, i.e. proton halo or neutron halo. In Ref. [9], it is shown that the unexpected behavior of ${ }^{8} \mathrm{~B}+{ }^{58} \mathrm{Ni}$ gives a signature of different breakup dynamics effects for proton halo nuclei compared to neutron halo nuclei.

Hence, in this paper we extend our study for the reactions of weakly bound projectiles especially neutron halo nuclei like ${ }^{15} \mathrm{C}$ and ${ }^{17} \mathrm{~B}$ and proton halo nuclei like ${ }^{17} \mathrm{~F}$ and ${ }^{17} \mathrm{Ne}$ with a ${ }^{209} \mathrm{Bi}$ target.

In Table 3, matter radii calculated for the neutron halo nuclei ${ }^{15} \mathrm{C}$ and ${ }^{17} \mathrm{~B}$ and proton halo nuclei ${ }^{17} \mathrm{~F}$ and ${ }^{17} \mathrm{Ne}$ are displayed along with experimentally measured values. It is clear from the table 


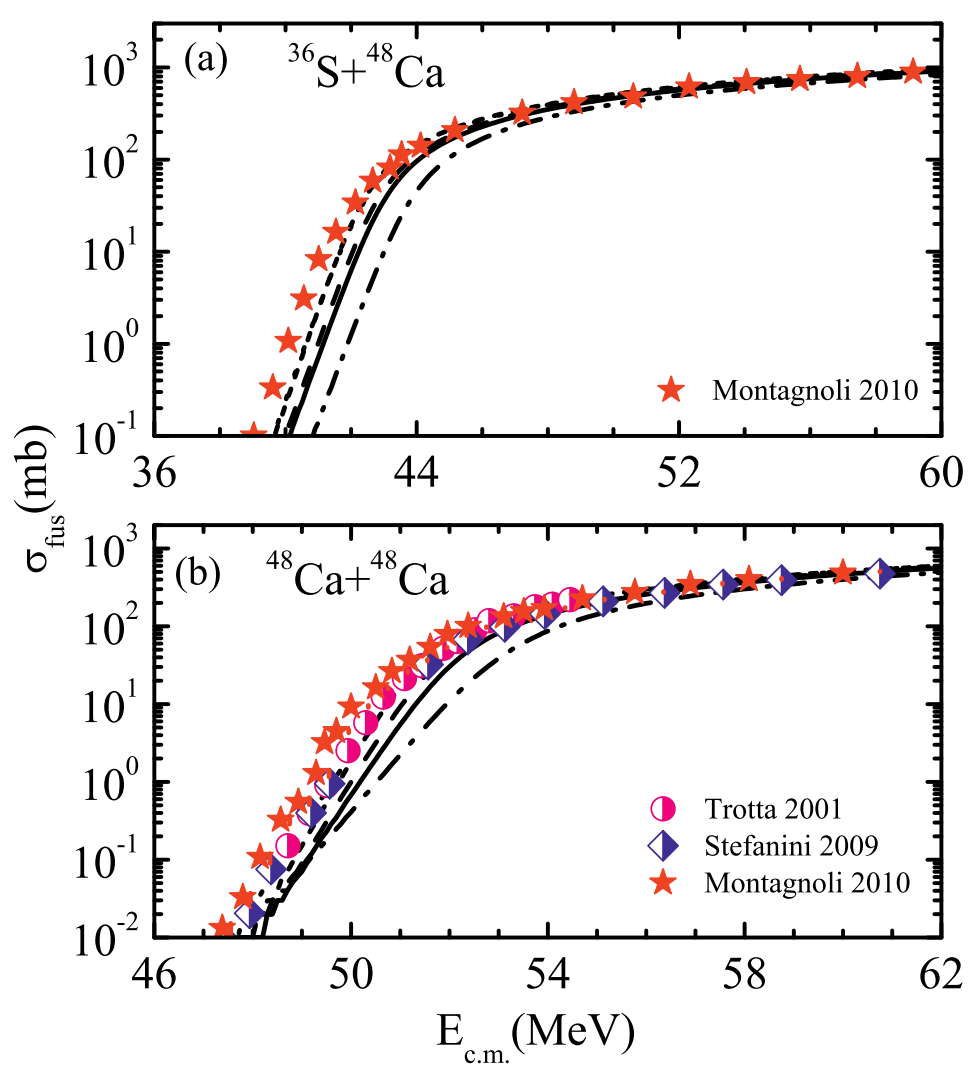

Figure 5. Same as fig. 2, but for the reactions of ${ }^{36} \mathrm{~S}+{ }^{48} \mathrm{Ca}$ and ${ }^{48} \mathrm{Ca}+{ }^{48} \mathrm{Ca}$. The experimental data are taken from Montagnoli 2010 [24], Trotta 2001 [25] and Stefanini 2009 [26].

that the matter radii calculated using these proximity based potentials are either lower or higher than the measured values. We are interested to see the effect of including the experimentally measured nuclear radii on fusion barrier heights and fusion probabilities using proximity based potentials. Since SEDF is applicable to even-even nuclei and Denisov DP is used for nuclei with $A \geq 16$, we restrict our attention to the potentials AW 95, Bass 80 and Proximity 2010. Table 4 gives barrier heights calculated using these proximity potentials.

In Figs. 6-8, we display the reduced fusion excitation functions $\sigma_{\text {reduced }}(\mathrm{mb})$ calculated using the Wong formula as a function of the reduced center of mass energy, $E_{\text {reduced }}(\mathrm{MeV})$. The solid, dashed and dash-dotted black lines represent the calculations for the AW 95, Bass 80 and Proximity 2010 potentials, respectively. The solid, dashed and dash-dotted gray lines represent calculations for the AW $95^{\text {halo }}$, Bass $80^{\text {halo }}$ and Proximity $2010^{\text {halo }}$ potentials, respectively (including experimentally measured radii). Here, Fig. 6 is a part of Fig. 2 of Ref. [10]. In this figure, we notice fusion suppression above the barrier and enhancement at sub-barrier energies in the case of ${ }^{6} \mathrm{He}+{ }^{209} \mathrm{Bi}$, as predicted by Gomes and collaborators [8] but enhancement throughout the energy range for the reaction of ${ }^{8} \mathrm{~B}+{ }^{58} \mathrm{Ni}$. We also found that the fusion cross sections calculated using the AW $95^{\text {halo }}$, 
Table 3. The calculated matter radii of proton halo and neutron halo projectiles and those from cross-section measurements. The experimentally measured values are also listed.

\begin{tabular}{|c|c|c|c|c|c|}
\hline \multirow[t]{2}{*}{$\overline{\text { Nucleus }}$} & \multicolumn{3}{|c|}{ "Nuclear radii(fm) } & \multirow[b]{2}{*}{ Expt. } & \multirow[b]{2}{*}{ Ref. } \\
\hline & AW 95 & Bass 80 & Prox 2010 & & \\
\hline \multicolumn{6}{|c|}{ Neutron halo } \\
\hline${ }^{6} \mathrm{He}$ & 2.09 & 2.01 & 1.78 & 2.71 & {$[28]$} \\
\hline${ }^{15} \mathrm{C}$ & 2.87 & 2.72 & 2.35 & 2.50 & [29] \\
\hline${ }^{17} \mathrm{~B}$ & 3.00 & 2.84 & 2.48 & 2.90 & [29] \\
\hline \multicolumn{6}{|c|}{ Proton halo } \\
\hline${ }^{8} \mathrm{~B}$ & 2.31 & 2.20 & 1.97 & 2.50 & {$[28]$} \\
\hline${ }^{17} \mathrm{~F}$ & 3.00 & 2.84 & 2.52 & 3.70 & [30] \\
\hline${ }^{17} \mathrm{Ne}$ & 3.00 & 2.84 & 2.56 & 2.75 & [29] \\
\hline
\end{tabular}

Table 4. The calculated barrier heights for the reactions of weakly bound projectiles with heavy targets using proximity based potentials and those obtained by including the extended matter radii in these potentials.

\begin{tabular}{ccccccc}
\hline \hline Reaction & $\begin{array}{c}\text { AW 95 } \\
V_{B} \\
(\mathrm{MeV})\end{array}$ & $\begin{array}{c}\text { AW 95 halo } \\
V_{B} \\
(\mathrm{MeV})\end{array}$ & $\begin{array}{c}\text { Bass } 80 \\
V_{B} \\
(\mathrm{MeV})\end{array}$ & $\begin{array}{c}\text { Bass 80 } \\
V_{B} \\
(\mathrm{MeV})\end{array}$ & $\begin{array}{c}\text { Prox 2010 } \\
V_{B} \\
(\mathrm{MeV})\end{array}$ & $\begin{array}{c}\text { Prox 2010 halo } \\
V_{B} \\
(\mathrm{MeV})\end{array}$ \\
\hline \hline Neutron halo & & & & & \\
\hline${ }^{6} \mathrm{He}+{ }^{209} \mathrm{Bi}$ & 19.95 & 18.66 & 19.51 & 17.77 & 19.32 & 17.47 \\
${ }^{15} \mathrm{C}+{ }^{209} \mathrm{Bi}$ & 57.73 & 60.15 & 56.93 & 58.62 & 58.26 & 57.30 \\
${ }^{17} \mathrm{~B}+{ }^{209} \mathrm{Bi}$ & 47.12 & 47.60 & 46.20 & 45.58 & 47.34 & 45.32 \\
\hline${ }^{\text {Proton halo }}$ & & & & & & \\
${ }^{8} \mathrm{~B}+{ }^{58} \mathrm{Ni}$ & 21.10 & 20.54 & 21.01 & 19.93 & 20.61 & 19.14 \\
${ }^{17} \mathrm{~F}+{ }^{209} \mathrm{Bi}$ & 87.02 & 81.05 & 86.51 & 78.55 & 87.96 & 78.09 \\
${ }^{17} \mathrm{Ne}+{ }^{209} \mathrm{Bi}$ & 97.12 & 99.79 & 96.88 & 98.07 & 97.96 & 95.91 \\
\hline \hline
\end{tabular}




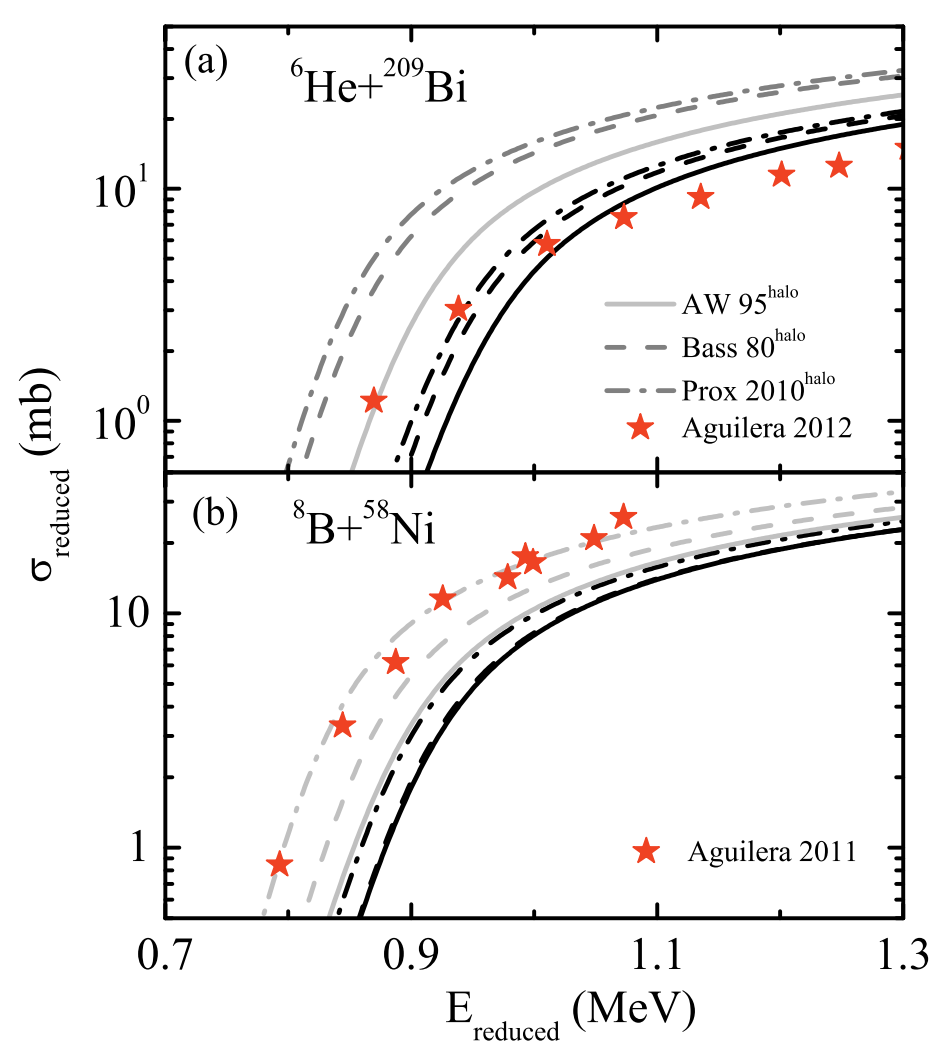

Figure 6. The reduced fusion cross sections $\sigma_{\text {reduced }}(\mathrm{mb})$ for the reactions of ${ }^{6} \mathrm{He}+{ }^{209} \mathrm{Bi}$ and ${ }^{8} \mathrm{~B}+{ }^{58} \mathrm{Ni}$ as a function of reduced center of mass energies $\mathrm{E}_{\text {reduced }}(\mathrm{MeV})$. The experimental data are taken from Aguilera 2012 [31] and Aguilera 2011 [32]. The different lines are explained in the text.

Bass $80^{\text {halo }}$ and Proximity $2010^{\text {halo }}$ potentials for the reactions of ${ }^{6} \mathrm{He}+{ }^{209} \mathrm{Bi},{ }^{11} \mathrm{Be}+{ }^{209} \mathrm{Bi}$ and ${ }^{8} \mathrm{~B}+$ ${ }^{58} \mathrm{Ni}$ increase on including the halo radii. However, in the present case, only Proximity $2010^{\text {halo }}$ gives enhanced fusion cross sections for all the considered reactions. Bass $80^{\text {halo }}$ shows enhancement in fusion cross sections in the cases of ${ }^{17} \mathrm{~B}$ and ${ }^{17} \mathrm{~F}$ and $\mathrm{AW} 95^{\text {halo }}$ gives enhanced an fusion cross section in the case of ${ }^{17} \mathrm{~F}$ only. Since the trend for fusion cross sections (on including halo radii) of these weakly bound nuclei is not clear, more experiments are invited for these reactions in order to compare with our theoretical calculations.

\section{Summary}

We have performed calculations to predict the fusion cross sections of the reactions of tightly bound as well as weakly bound projectiles with heavy targets. In the case of reactions of tightly bound projectiles, calculations using proximity based potentials are found to be in close agreement with experimental data. In the case of reactions involving weakly bound projectiles, especially halo nuclei, 


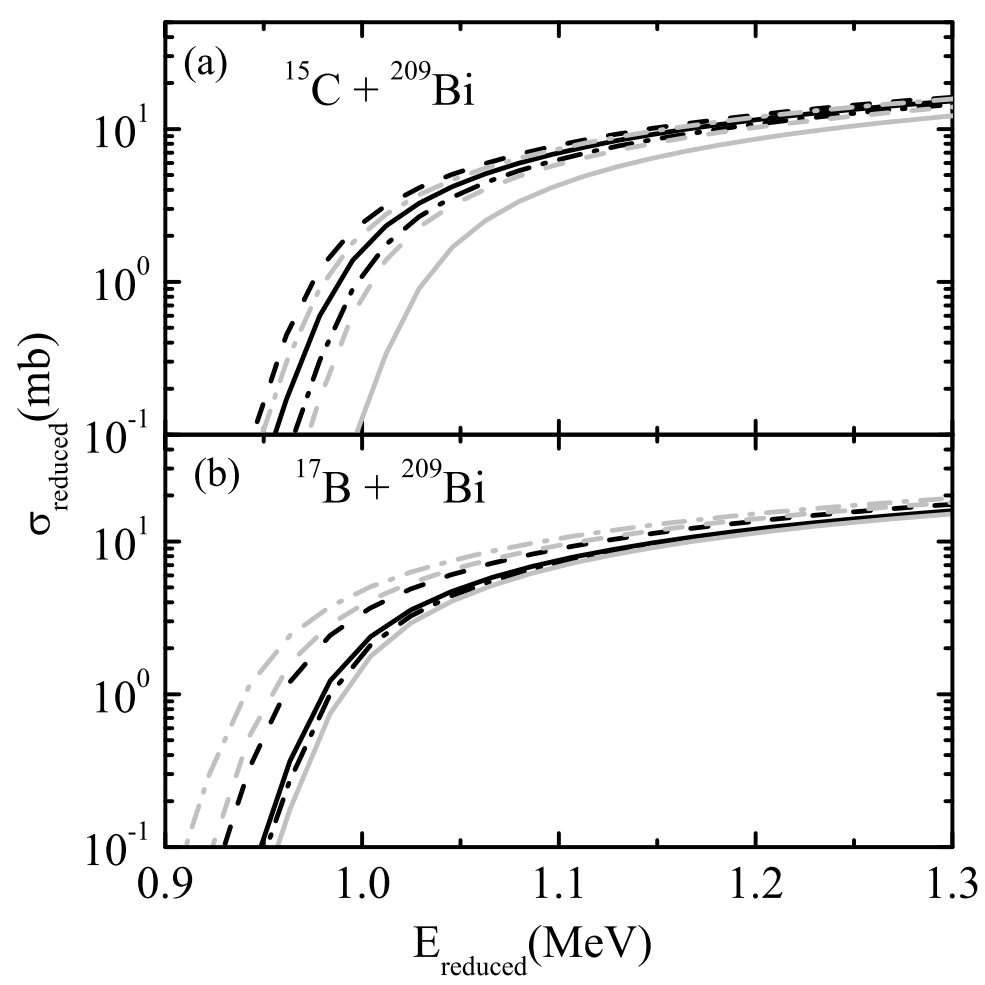

Figure 7. Same as fig. 6, but for the reactions of ${ }^{15} \mathrm{C}+{ }^{209} \mathrm{Bi}$ and ${ }^{17} \mathrm{~B}+{ }^{209} \mathrm{Bi}$.

the extended radii significantly affect barrier heights as well as fusion probabilities. The observed large enhancement throughout the energy range in the fusion cross sections of the proton halo projectile ${ }^{17} \mathrm{~F}$ (see ${ }^{17} \mathrm{~F}+{ }^{209} \mathrm{Bi}$ reaction) is an important question for further experimental study. Since fusion cross sections of very few proton halo nuclei has been measured till now, it is very important to investigate experimentally the reactions of these weakly bound nuclei with a ${ }^{209} \mathrm{Bi}$ target.

\section{Acknowledgedment}

The financial supports from the Council of Scientific and Industrial Research (CSIR), New Delhi, and CNR*13 are appreciatively acknowledged. The author acknowledges collaboration with Professor Rajeev K. Puri and Dr. Ishwar Dutt. 


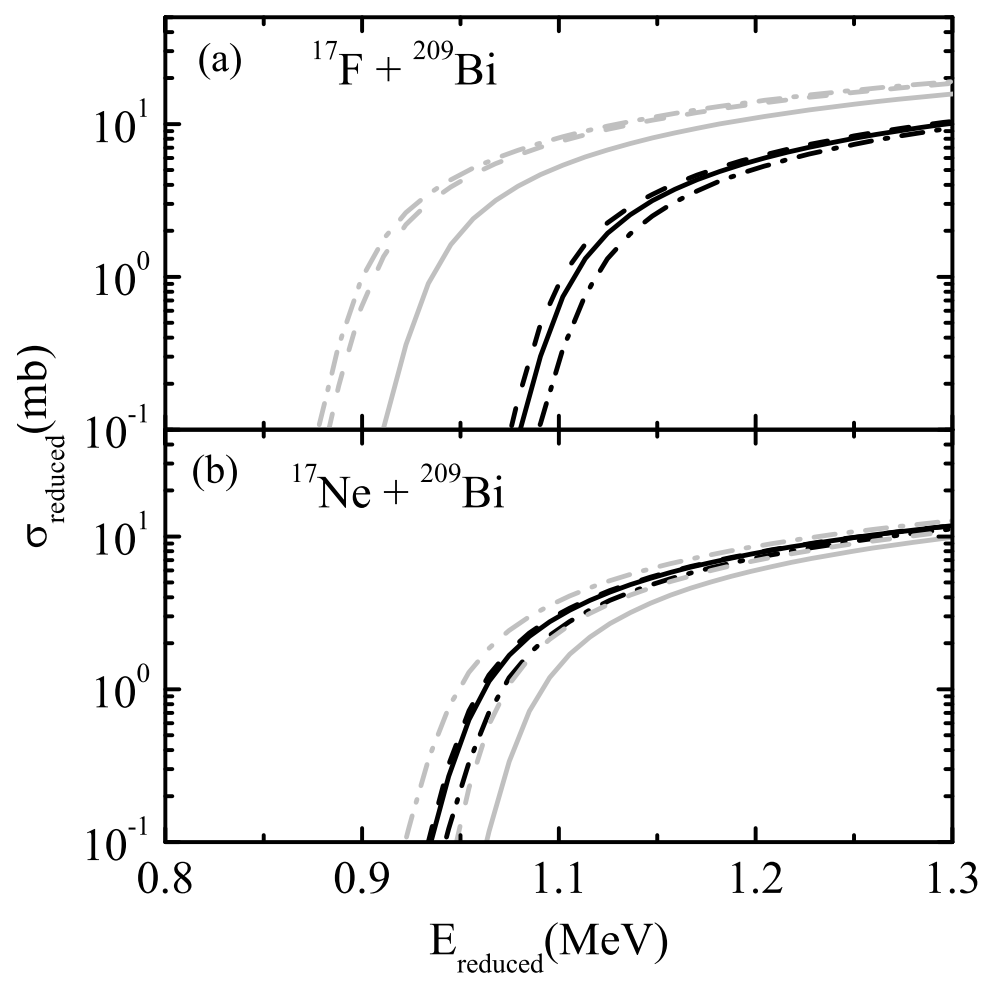

Figure 8. Same as fig. 6, but for the reactions of ${ }^{17} \mathrm{~F}+{ }^{209} \mathrm{Bi}$ and ${ }^{17} \mathrm{Ne}+{ }^{209} \mathrm{Bi}$.

\section{References}

[1] L. C. Vaz, J. M. Alexander and G. R. Satchler, Phys. Rep. 69, 373 (1981).

[2] Y. K. Vermani and R. K. Puri, Eur. Phys. Lett. 85, 62001 (2009); ibid. J. Phys. G: Nucl. Part. Phys. 36, 105103 (2009); Y. K. Vermani, S. Goyal and R. K. Puri, Phys. Rev. C 79, 064613 (2009); Y. K. Vermani et al., J. Phys. G: Nucl. Part. Phys. 37, 015105 (2010); S. Goyal and R. K. Puri, Phys. Rev. C 83, 047601 (2011); S. Kaur and R. K. Puri, Phys. Rev. C 87, 014620 (2013).

[3] S. Kumar et al., Phys. Rev. C 58, 3494 (1998); S. Gautam et al., J. Phys. G: Nucl. Phys. 37, 085102 (2010); S. Gautam et al., Phys. Rev. C 83, 014603 (2011); ibid. C 83, 034606 (2011); ibid. C 85, 067601 (2012); ibid. C 86, 034607 (2012); R. Bansal et al., Phys. Rev. C 87, 01601 (2013).

[4] Y. Yano, Nucl. Instrum. and Methods B 261, 1009 (2007).

[5] J. J. Kolata et al., Phys. Rev. C 85, 054603 (2012); A. Pakou et al., Phys. Rev. C 87, 0146193 (2013); G. Montagnoli et al., Phys. Rev. C 85, 024607 (2012).

[6] V. V. Sargsyan et al., Phys. Rev. C 86, 054610 (2012); P. R. S. Gomes et al., Phys. Lett. B 695, 320 (2011).

[7] A. B. Balantekin and N. Takigawa, Rev. Mod. Phys. 70, 77 (1998). 
[8] P. R. S. Gomes et al., J. Phys. G: Nucl. Phys. 39, 115103 (2012); ibid. J. Phys.: Conf. Ser. 420, 012121 (2013).

[9] J. Rangel et al., Eur. Phys. J. A 49, 57 (2013).

[10] R. Kumari, Nucl. Phys. A 917, 85 (2013).

[11] I. Dutt and R. K. Puri, Phys. Rev. C 81, 044615 (2010); ibid. C 81, 064609 (2010); ibid. C 81, 047601 (2010); ibid. C 81, 064608 (2010).

[12] I. Dutt and R. Bansal, Chin. Phys. Lett. 27, 112402 (2010).

[13] R. K. Puri and R. K. Gupta, Phys. Rev. C 45, 1837 (1992); ibid. C 51, 1568 (1995); M. K. Sharma et al., Eur. Phys. J. A 2, 69 (1998); ibid. A 3, 277 (1998); R. K. Puri, R. Arora and R. K. Gupta, Phys. Rev. C 60, 054619 (1999); ibid. Eur. Phys. J. A 8, 107 (2000).

[14] R. Bass, . Phys. A 231, 45 (1974); R. K. Puri and N. Dhiman, Eur. Phys. J. A 23, 429 (2005); ibid. Acta Physica Polonica B 37, 1855 (2006); ibid. B 38, 2133 (2007).

[15] V. Yu. Denisov, Phys. Lett. B 526, 315 (2002).

[16] A. Winther, Nucl. Phys. A 594, 203 (1995).

[17] J. Blocki, J. Randrup, W. J. Swiatecki, and C. F. Tsang, Ann. Phys. 105, 427 (1977).

[18] R. K. Puri, P. Chattopadhyay and R. K. Gupta, Phys. Rev. C 43, 315 (1991).

[19] C. Y. Wong, Phys. Lett. B 32, 567 (1970); ibid. B 42, 186 (1972); C. Y. Wong, Phys. Rev. Lett. 31, 766 (1973).

[20] E. F. Aguilera and J. J. Kolata, Phys. Rev. C 85, 014603 (2012).

[21] A. M. Stefanini et al., Nucl. Phys. A 456, 509 (1986).

[22] K. Siwek-Wilczy'nska and J. Wilczy'nski, Phys. Rev. C 69, 024611 (2004).

[23] A. M. Stefanini et al., Phys. Rev. C 78, 044607 (2008).

[24] G. Montagnoli et al., Phys. Rev. C 82, 064609 (2010).

[25] M. Trotta et al., Phys. Rev. C 65, 011601 (R) (2001).

[26] A. M. Stefanini et al., Phys. Lett. B 679, 95 (2009).

[27] J. O. Newton et al., Phys. Rev. C 70, 024605 (2004).

[28] J. S. Al-Khalili, J. A. Tostevin and I. J. Thompson, Phys. Rev. C 54, 1843 (1996).

[29] K. Riisager, Phys. Scr. T 152, 014001 (2013).

[30] J. F. Liang et al., Phys. Rev. C 65, 051603 (2002).

[31] E. F. Aguilera and J. J. Kolata, Phys. Rev. C 85, 014603 (2012).

[32] E. F. Aguilera et al., Phys. Rev. Lett. 107, 092701 (2011). 